\title{
Subdural Hematoma Due to Ruptured Intracranial Aneurysm
}

\author{
Kazuko Kamiya, Tetsuji Inagawa, Mitsuo Yamamoto* \\ and Shuji MONDEN** \\ Department of Neurosurgery, Shimane Prefectural Central Hospital, Izumo, Shimane; \\ *Department of Neurosurgery, Matsue Red Cross Hospital, Matsue; \\ **Department of Neurosurgery, Onomichi General Hospital, Onomichi, Hiroshima
}

\begin{abstract}
Subdural hematoma (SDH) was observed in 15 of 484 cases of aneurysmal subarachnoid hemorrhage (SAH). There were four males and 11 females, with ages ranging from 39 to 75 years. The clinical grades (Hunt and Hess) on admission were II in three cases, III in two, IV in four, and V in six. The ruptured aneurysms were located in the middle cerebral artery (MCA) in six cases, anterior communicating artery in three, internal carotid artery in two, and distal anterior cerebral artery (ACA) in two, with two cases unconfirmed. A high proportion of aneurysms occurred in the MCA and distal ACA. Aneurysmal neck clipping and removal of SDH were performed in the acute stage of seven cases, without intraoperative rerupture. The outcomes 1 year after SAH of the seven patients undergoing surgery were good recovery in five, but in two, vegetative state due to preoperative rerupture or medical complications. All eight patients without surgical intervention died. A good prognosis for patients with ruptured intracranial aneurysms accompanied by SDH can be expected with direct surgical intervention in the acute stage, even if the clinical grade on admission is poor.
\end{abstract}

Key words: intracranial aneurysm, subarachnoid hemorrhage, subdural hematoma

\section{Introduction}

Subarachnoid hemorrhage (SAH) almost invariably develops following the rupture of a cerebral aneurysm, and is often complicated by intracerebral hematoma $(\mathrm{ICH})$ and intraventricular hematoma (IVH). But only extremely rarely does subdural hematoma (SDH) occur. The clinical incidence of $\mathrm{SDH}$ complications is reported to be $0.5-7.9 \% .^{2,3,12)}$ In the present study, the clinical grade on admission, location and size of the ruptured aneurysm, and outcome were examined in cases of SDH.

\section{Materials and Methods}

Between January of 1979 and December of 1988, a total of 484 cases of SAH due to ruptured intracranial aneurysms were admitted to the Department of Neurosurgery, Shimane Prefectural Central
Hospital. In 15 cases, SDH was demonstrated by computed tomography (CT). The clinical grade on admission, site, size, and presence or absence of bleb of ruptured aneurysms, and the outcome of the 15 cases with SDH and 469 cases without SDH were compared. The clinical grade on admission was assessed by the Hunt and Hess scale. ${ }^{6}$ The outcome was evaluated using the Glasgow Outcome Scale? 1 year after SAH.

\section{Results}

CT scanning was conducted on 452 of the 484 cases within 14 days of the aneurysmal rupture. The CT findings are summarized in Table 1. SDH was observed in 15 cases, and their clinical features are summarized in Table 2.

Of the 469 cases without SDH, the clinical grades on admission were I in $53(11 \%)$, II in $177(38 \%)$, III

Received April 2, 1990; Accepted June 20, 1990

Author's present address: K. Kamiya, M.D., Statistics and Information Department, Ministry of Health and Welfare, Tokyo, Japan. 
Table 1 CT findings in 452 cases with aneurysmal $\mathrm{SAH}^{*}$

\begin{tabular}{lr} 
CT findings & No. of cases \\
\cline { 1 - 1 } SAH only & 307 \\
ICH & 109 \\
IVH & 86 \\
SDH & 15
\end{tabular}

${ }^{*}$ No CT scan was performed in the acute stage of 32 cases.

in $110(23 \%)$, IV in $56(12 \%)$, and $\mathrm{V}$ in $73(16 \%)$. Among the 15 cases with SDH, the clinical grades were II in three $(20 \%)$, III in two $(13 \%)$, IV in four $(27 \%)$, and $V$ in six $(40 \%)$, with the majority of cases belonging to the poorer grades $(\mathrm{p}<0.05)$. Eighty$\operatorname{six}(18 \%)$ of 469 non-SDH patients were paretic on admission. The frequency of paresis was higher among the SDH patients, in six $(40 \%)$ of 15 cases $(\mathrm{p}<0.05)$.

The initial CT scans of the $15 \mathrm{SDH}$ cases showed $\mathrm{SDH}+\mathrm{SAH}$ in four, $\mathrm{SDH}+\mathrm{SAH}+\mathrm{ICH}$ in six, $\mathrm{SDH}+\mathrm{SAH}+\mathrm{ICH}+\mathrm{IVH}$ in four, and $\mathrm{SDH}$ only in one. The SDH was observed after initial rupture in 10 cases and after rerupture in five.

The location of ruptured aneurysms could be confirmed in 427 of 469 non-SDH cases. The sites were the anterior communicating artery (AcomA) in 164
Table 3 Maximum diameter of ruptured aneurysms*

\begin{tabular}{ccc}
\cline { 2 - 3 } Diameter $(\mathrm{mm})$ & With SDH & Without SDH \\
\hline$<5$ & $5(38 \%)$ & $116(27 \%)$ \\
$<10$ & $3(23 \%)$ & $216(51 \%)$ \\
$<20$ & $1(8 \%)$ & $79(19 \%)$ \\
$<25$ & $1(8 \%)$ & $1(0.2 \%)$ \\
$\geqq 25$ & $3(23 \%)$ & $15(4 \%)$ \\
\hline Total & 13 & 427 \\
\hline
\end{tabular}

${ }^{*}$ Ruptured aneurysms were not measured in two cases with SDH and in 42 without SDH.

cases $(38 \%)$, internal carotid artery (ICA) in 117 $(27 \%)$, middle cerebral artery (MCA) in $101(24 \%)$, distal anterior cerebral artery (ACA) in $26(6 \%)$, and vertebrobasilar artery in $19(4 \%)$. The ruptured aneurysms could be confirmed in 13 of $15 \mathrm{SDH}$ cases, being the AcomA in three (23\%), ICA in two $(15 \%)$, MCA in six $(46 \%)$, and distal ACA in two $(15 \%)$. Therefore, a high proportion of aneurysms occur in the MCA and distal ACA. The presence or absence of the bleb of aneurysm showed no definite trend. Larger or giant aneurysms were relatively frequent in the 13 cases with SDH $(p<0.01$ ) (Table 3 ).

Neck clipping and removal of SDH were performed during the acute stage in seven of the 15 SDH patients. No ruptures occurred during the surgical pro-

Table 2 Clinical summary of 15 patients with SDH due to ruptured intracranial aneurysm

\begin{tabular}{|c|c|c|c|c|c|c|c|c|c|c|c|}
\hline \multirow{2}{*}{$\begin{array}{l}\text { Case } \\
\text { No. }\end{array}$} & \multirow{2}{*}{$\begin{array}{l}\text { Age/ } \\
\text { Sex }\end{array}$} & \multirow{2}{*}{ Grade* } & \multirow{2}{*}{ Paresis } & \multicolumn{3}{|c|}{ Other CT findings } & \multirow[b]{2}{*}{ Site } & \multicolumn{2}{|c|}{ Aneurysm } & \multirow{2}{*}{$\begin{array}{c}\text { Direct } \\
\text { operation }\end{array}$} & \multirow{2}{*}{ Outcome** } \\
\hline & & & & $\overline{\mathrm{SAH}}$ & $\mathrm{ICH}$ & IVH & & Bleb & Diameter (mm) & & \\
\hline 1 & $67 / F$ & IV & + & + & - & - & MCA & + & 30 & + & VS \\
\hline 2 & $50 / \mathrm{F}$ & III & - & + & + & + & ICA & - & 28 & - & D \\
\hline 3 & $67 / F$ & $\mathrm{~V}$ & + & + & + & - & $\mathrm{MCA}$ & - & 4 & - & $\mathrm{D}$ \\
\hline 4 & $52 / \mathrm{F}$ & $\mathrm{V}$ & - & + & + & + & ND & & & - & $\mathrm{D}$ \\
\hline 5 & $69 / \mathrm{F}$ & II & - & + & + & + & AcomA & + & 27 & - & $\mathrm{D}$ \\
\hline 6 & $63 / \mathrm{F}$ & II & - & + & - & - & MCA & - & 4 & + & GR \\
\hline 7 & $73 / \mathrm{M}$ & IV & + & + & - & - & $A \operatorname{com} A$ & + & 7 & - & $\mathrm{D}$ \\
\hline 8 & $75 / \mathrm{F}$ & II & - & $+\#$ & - & - & $\mathrm{MCA}$ & + & 9 & + & VS \\
\hline 9 & $64 / M$ & $\mathrm{~V}$ & - & + & - & - & ND & & & - & $\mathrm{D}$ \\
\hline 10 & $72 / F$ & IV & + & + & + & - & $\underset{\mathrm{ACA}}{\text { distal }}$ & - & 4 & + & GR \\
\hline 11 & $70 / \mathrm{M}$ & IV & + & + & + & - & $\mathrm{MCA}$ & + & 6 & + & GR \\
\hline 12 & $72 / F$ & $\mathrm{~V}$ & - & + & + & - & ICA & - & 4 & - & $\mathrm{D}$ \\
\hline 13 & $59 / \mathrm{M}$ & $\mathrm{V}$ & - & + & + & + & $\mathrm{MCA}$ & + & 22 & - & $\mathrm{D}$ \\
\hline 14 & $39 / \mathrm{F}$ & $\mathrm{V}$ & + & + & + & - & $\begin{array}{l}\text { distal } \\
\text { ACA }\end{array}$ & - & 3 & + & GR \\
\hline 15 & $71 / F$ & III & - & + & + & - & Acom $A$ & + & 11 & + & GR \\
\hline
\end{tabular}

${ }^{*}$ Hunt and Hess grade $\left.{ }^{6}\right)$ without modification on admission. ${ }^{* *}$ Glasgow Outcome Scale ${ }^{7} 1$ year after initial SAH. GR: good recovery, MD; moderate disability, SD: severe disability, VS: vegetative state, D: died. "Absent on initial CT. ND: not detected. 
Table 4 Outcome 1 year after aneurysmal rupture*

\begin{tabular}{cccrrr}
\multirow{2}{*}{ Outcome $^{* *}$} & \multicolumn{2}{c}{ With SDH } & & \multicolumn{2}{c}{ Without SDH } \\
\cline { 2 - 3 } & Surgery $(+)$ & $(-)$ & $(+)$ & $(-)$ \\
GR & 5 & 0 & 206 & 27 \\
MD & 0 & 0 & 31 & 0 \\
SD & 0 & 0 & 29 & 3 \\
VS & 2 & 0 & 9 & 3 \\
D & 0 & 8 & 38 & 113 \\
\hline Total & 7 & 8 & 313 & 146
\end{tabular}

*Ten cases without SDH did not survive 1 year after aneurysmal rupture. ${ }^{* *}$ Glasgow Outcome Scale. ${ }^{7)}$

cedure. The outcomes of five patients were good, but one (Case 1) developed decubitus and pneumonia and another (Case 8 ) became vegetative in association with preoperative rerupture and cardiac failure. However, none of the eight patients without surgery have survived. Two patients were inoperable because of rerupture or severe spasm on admission. Five of six patients in grade $\mathrm{V}$ on admission were inoperable, and in one of four patients in grade IV on admission, clinical grade became aggravated to grade $\mathrm{V}$ and the operation was impossible. Only one patient in grade $\mathrm{V}$ received surgical intervention and survived. The outcomes of the 459 non-SDH patients surviving more than 1 year were good recovery in 233 $(51 \%)$, moderate disability in $31(7 \%)$, severe disability in $32(7 \%)$, vegetative state in $12(3 \%)$, and death in $151(33 \%)$ (Table 4$)$.

\section{Case Reports}

Case 8: A 75-year-old female was admitted on August 20,1985, because of severe headache. Her consciousness was almost clear. The initial CT scans on admission showed only SDH and not SAH, suggesting chronic SDH (Fig. 1 upper). Burr-hole irrigation was therefore performed, but fresh hematoma was observed under the dura mater. She became unconscious while returning from the operating room. Repeat CT scans revealed SAH with SDH (Fig. 1 lower). Right carotid angiograms revealed an MCA aneurysm. Complications of pulmonary edema and cardiac failure appeared at the same time. Aneurysmal neck clipping and SDH removal were performed on the 2 nd day. One year later, she continued to be vegetative.

Case 14: A 39-year-old female was admitted on April 17,1988 , because of a sudden loss of consciousness. On admission, she was in a deep coma with decerebrate posturing, absent pupillary light reflex
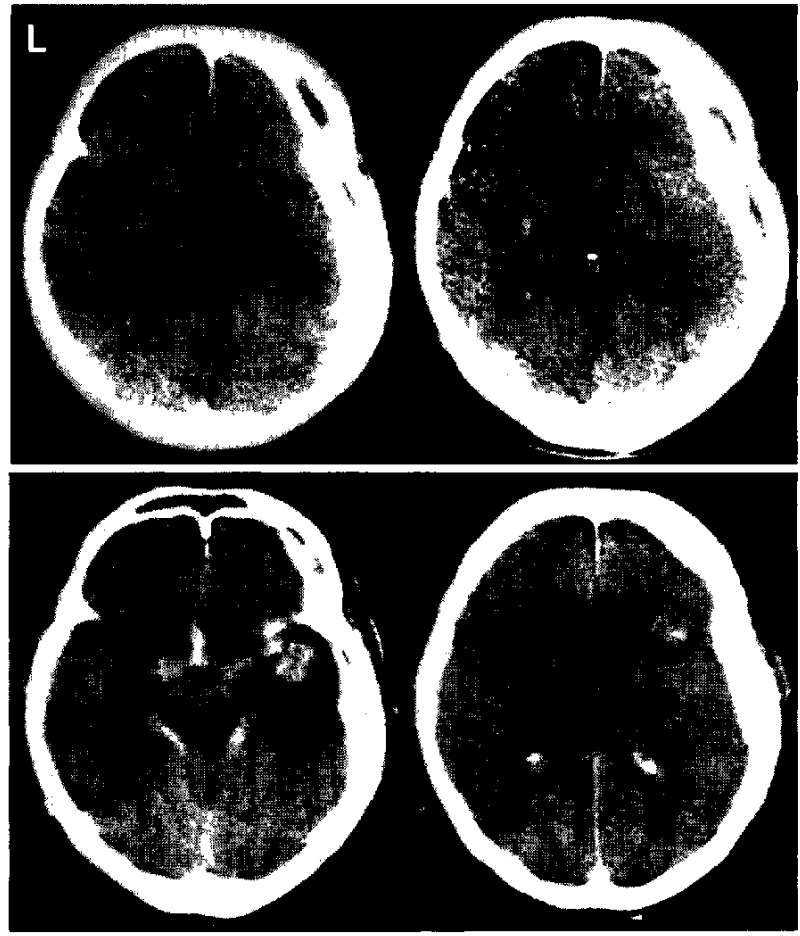

Fig. 1 Case 8. upper: CT scans on admission, showing SDH but not SAH. lower: CT scans after aggravation, demonstrating $\mathrm{SAH}$ with $\mathrm{SDH}$.

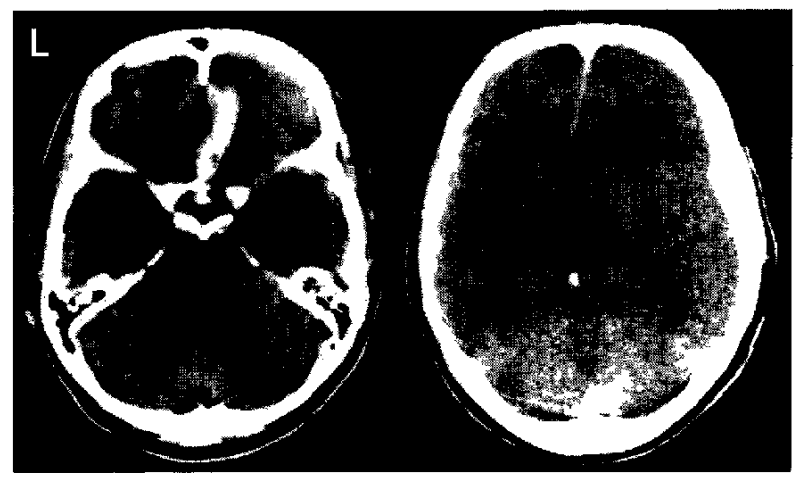

Fig. 2 Case 14. CT scans on admission, showing $\mathrm{SAH}, \mathrm{ICH}$, and SDH with the midline shift.

on the right, and anisocoria. CT scans revealed $\mathrm{SAH}, \mathrm{ICH}$, and SDH with a midline shift (Fig. 2). Right carotid angiograms revealed a distal ACA aneurysm. Aneurysmal neck clipping and removal of the SDH were performed the same day. One year after the onset, she was in good health. 


\section{Discussion}

Clarke and Walton ${ }^{4)}$ classified SDH due to ruptured intracranial aneurysms into two groups based on the path of the blood from the ruptured aneurysm into the subdural space: by bleeding directly into the subdural space and bleeding into the subdural space via the subarachnoid space. Barton and Tudor ${ }^{21}$ described four mechanisms by which blood from a leaking aneurysm can reach the subdural space. 1) Successive small hemorrhages allow adhesions to develop, and the final rupture occurs into the subdural space. 2) The arachnoid membrane is ruptured by the rapid accumulation of blood under pressure from the leaking aneurysm. 3) A massive hemorrhage ruptures the cortex and lacerates the arachnoid membrane. 4) An aneurysm in the part of the carotid within the subdural space ruptures and directly causes SDH.

The confirmation of the presence or absence of successive small hemorrhages as in 1) is difficult. However, warning signs such as headache may reflect their presence. ${ }^{21}$ In the present study, three of 15 cases had such episodes. Mechanism 2) is said to be the commonest, but is difficult to differentiate completely from 1). As complications such as $\mathrm{ICH}$ are common in SDH cases, 3 ) is quite plausible. In the present study, associated SDH and ICH complications were very frequent, in 10 of 15 cases. In Cases 10 and 14, an ICH within the frontal lobe caused by the rupture of a distal ACA aneurysm penetrated the interhemispheric fissure and entered the subdural space. In Case 11 , the $\mathrm{ICH}$ due to rupture of a MCA aneurysm was extremely large, flowing into the Sylvian cistern and further extending to the subdural space. However, the AcomA aneurysm in Case 15 was confined to the frontal lobe, and no associated SDH occurred. The SAH itself was severe in only four cases (Cases 2, 3, 7, and 12), moderate in five, slight in five, and absent in one. SDH does not therefore necessarily occur with massive hemorrhage into the subarachnoid space. Mechanism 4) has yet to be confirmed, and is considered unlikely by some authors. ${ }^{8)}$

An aneurysm at any site can cause a SDH upon rupture. ${ }^{10)}$ The rupture of distal ACA or AcomA aneurysms is rare, and that of ICA and MCA aneurysms common. ${ }^{1,5,12)}$ In the present study, the ruptures of MCA and distal ACA aneurysms were relatively frequent, observed in six cases and two, respectively.

SDH but not SAH was observed on admission of Case 8 with a MCA aneurysm, resulting in a wrong diagnosis. The delay in controlling the systemic blood pressure caused rerupture of the aneurysm, which aggravated the clinical grade to IV. As frequently emphasized in the past, this is a case demonstrating clearly the grave probability that SDH has developed from a ruptured intracranial aneurysm. ${ }^{1,8,9)}$

Surgical removal of SDH in the acute stage involves the risk of rupture due to rapid decompression. However, some recent reports have suggested that aneurysmal neck clipping and SDH removal by extensive craniotomy can be conducted simultaneously. ${ }^{1}$ In all our cases, surgery in the acute stage caused no ruptures.

Previous authors have pointed out that in the majority of SDH cases due to ruptured aneurysm the clinical grades are severe. ${ }^{8,11}$ In six of the 15 present cases, the clinical grade was V. Case 4 was dead on arrival, and Case 9 was in the non-filling state during cerebral angiography. Cases 3,12 , and 13 died on days 0,1 , and 2 , respectively. However, surgery conducted on Case 14 (grade V) during the acute stage resulted in good recovery. Extremely favorable results have been observed in SDH cases with poor clinical grades on admission. The $\mathrm{SAH}$ was minimal in most cases, and the common causes of the poor clinical grade were increased intracranial pressure due to SDH and the midline shift. Even if the clinical grade is poor, in cases of mild SAH the delayed ischemic neurological deficiency due to cerebral vasospasm may be small. ${ }^{11}$ As SDH is a hematoma caused by blood flow from the subarachnoid or intracranial space, the brain damage is smaller than that caused by trauma. Sachs and Bernat ${ }^{11)}$ have pointed out the necessity for prompt diagnosis using CT scans and mannitol treatment. In addition, positive steps should be taken to treat patients with ruptured aneurysms accompanied by SDH, even if the clinical grade on admission is poor.

\section{Acknowledgment}

The assistance of Miss Yoshiko liguni in the preparation of this manuscript is gratefully acknowledged.

\section{References}

1) Ban $S$, Sato $S$, Yamamoto $T$, Ogata $M$ : Distal anterior cerebral artery aneurysms causing acute subdural hematoma: Report of two cases. No Shinkei Geka 13: 911-916, 1985 (in Japanese)

2) Barton E, Tudor J: Subdural haematoma in association with intracranial aneurysm. Neuroradiology 23: $157-160,1982$

3) Bassett CR, Lemmen LF: Subdural hematoma as- 
sociated with bleeding intracranial aneurysm. $J$ Neurosurg 9: 443-450, 1952

4) Clarke E, Walton JN: Subdural haematoma complicating intracranial aneurysm and angioma. Brain 76: 378-404, 1953

5) Hirashima Y, Endo S, Horie Y, Koshu K, Takaku A: An anterior communicating artery aneurysm complicated by chronic subdural hematoma: A case report. No Shinkei Geka 9: 1041-1045, 1981 (in Japanese)

6) Hunt WE, Hess RM: Surgical risk as related to time of intervention in the repair of intracranial aneurysms. J Neurosurg 28: 14-20, 1968

7) Jennett B, Bond $M$ : Assessment of outcome after severe brain damage. A practical scale. Lancet 1: $480-484,1975$

8) Kondziolka D, Bernstein M, Brugge KT, Schutz H: Acute subdural hematoma from ruptured posterior communicating artery aneurysm. Neurosurgery 22: $151-154,1988$
9) O'Leary PM, Sweeny PJ: Ruptured intracerebral aneurysm resulting in a subdural hematoma. Ann Emerg Med 15: 944-946, 1986

10) Reynolds $A F$, Shaw CM: Bleeding patterns from ruptured intracranial aneurysms: An autopsy series of 205 patients. Surg Neurol 15: 232-235, 1981

11) Sachs E, Bernat JL: Recovery from acute subdural hematoma and uncal herniation due to ruptured intracranial aneurysm. Neurosurgery 3: 66-67, 1978

12) Strang RR, Tovi D, Hugosson R: Subdural hematomas resulting from the rupture of intracranial arterial aneurysms. Acta Chir Scand 121: 345-350, 1961

Address reprint requests to: K. Kamiya, M.D., Statistics and Information Department, Ministry of Health and Welfare, 7-3 Ichigaya Honmura-cho, Shinjukuku, Tokyo 162, Japan. 